\title{
sciendo
}

\section{BREAST MUSCLE MYOPATHIES IN BROILER: MECHANISM, STATUS AND THEIR IMPACT ON MEAT QUALITY - A REVIEW}

\author{
Ali Hassan Nawaz ${ }^{1}$, Jia Hui Zheng ${ }^{1}$, Wei Lu Zhang ${ }^{1}$, Fu Jian Wang ${ }^{1}$, Zheng Hai Jiao ${ }^{1}$, Kwaku Amoah², Li Zhang ${ }^{1 *}$ \\ ${ }^{1}$ College of Coastal Agricultural Sciences, Guangdong Ocean University, 524088, Zhanjiang, Guangdong, PR China \\ ${ }^{2}$ Aquatic Animals Precision Nutrition and High-Efficiency Feed Engineering Research Center of Guangdong Province, 524088, Zhanjiang, \\ Guangdong, PR China \\ •Corresponding author: zhangli761101@163.com
}

\begin{abstract}
Almost a decade ago, the sudden rise of breast muscle defects in fast-growing commercial broiler breeds challenged the broiler production industry and meat scientists to address the issue of these novel muscle abnormalities. After that, a widespread hypothesis showing a correlation between high muscle yield and incidence of these muscle myopathies received much acceptance from the research community. Increased muscle hypertrophy and unbalanced growth of connective tissues lead to an inadequate blood supply that ultimately causes hypoxia in muscle fibers. Reduced blood vascular density in muscle fibers induces oxidative stress and mitochondrial dysfunction, leading to muscle fibrosis, lipidosis and myodegeneration. Along with physical changes, the myopathic muscles exhibit poor sensory properties, abnormal texture properties and a low nutritional profile. As these myopathies alter meat's physical appearance, they have a negative impact on customer's behavior and preference. A better production environment with proper dietary supplementation with balanced breeding strategies can minimize the incidence of muscle myopathies in broiler chicken. This review aims to address the underlying mechanism behind these myopathies and their impact on poultry meat quality, including nutritional value and consumer behavior. It describes the link between genetic and non-genetic elements influencing myopathies, along with the strategies to minimize the occurrence of breast muscle myopathies.
\end{abstract}

Key words: breast muscle myopathies, oxidative stress, broiler production, meat quality, consumer behavior

In recent years, an increase in poultry meat demand over red meat has been observed in most parts of the world, as chicken meat is relatively cheap, nutritious, healthy, and easy to cook compared to other muscle foods (Bailey et al., 2020; Estévez, 2015; Wideman et al., 2016). To meet this ever-growing demand for poultry, it led to extensive genetic selection of broiler for optimum broiler production (Petracci et al., 2019). The poultry industry has achieved milestones over the previous 40 to 50 years regarding the feed conversion ratio and broiler chicken growth rates and that is the reason behind higher growth rate of commercial breeds as compared to the local breeds. Pushing these biological boundaries in terms of muscle gain has made the commercial chicken more vulnerable to certain meat myopathies. Commonly occurring myopathies include WS (white striping), WB (wooden breast), DPM (deep pectoral muscle myopathy) and PSE (pale, soft and exudative) meat. These myopathies occur in pectoral muscles and are closely linked to the rigorous and exhaustive poultry production system (Huang and Ahn, 2018; Petracci et al., 2019). These myopathies are a significant hindrance in consumer acceptance of poultry meat (de Carvalho et al., 2020). A study observed a decreasing trend in fresh meat consumption in European countries as chicken meat con- sumption decreased by 14\% from 2017 to 2018 in Spain (MAPA, 2018). It is suggested that modern intensive animal production systems are the main reason behind this consumer shift as it creates a sense of poor animal welfare and low meat quality (Bailey et al., 2020; EC, 2019; Skaperda et al., 2019).

It has been reported that chicken breast muscle myopathies occur worldwide, especially in countries where fast-growing commercial breeds are raised at large scale as the ratio of chicken breast abnormalities was relatively higher in commercial breeds than local chicken breeds (de Carvalho et al., 2020). WS, the most common of the myopathies, affects an overall level of $50 \%$ chicken breasts in Italy, France, Spain, and Brazil (Russo et al., 2015); out of those affected carcasses, $20-30 \%$ of muscles display severe damage. A study conducted on birds at nine weeks of age in the United States, showed that more than $98 \%$ displayed some signs of WS, out of which $55 \%$ were graded as moderate to severely affected, and $85 \%$ of the samples were affected by WB (42\% severe cases) (Kuttappan et al., 2017). Apart from age, the probability for occurrence of breast myopathies rises with high body weight at slaughter time and higher growth rate that supports the argument of genetic correlation of breast myopathies (Cruz et al., 2017; Lorenzi et al., 2014). Some histopathological stud- 
ies also pointed that myodegeneration process regarding these myopathies is strongly linked to the age of birds at slaughter (Kawasaki et al., 2018; Radaelli et al., 2017).

In most cases, the presence of white stripes along the direction of muscle fibers makes WS affected chicken breast easily recognizable that reduces consumer's satisfaction. Sometimes, it is regarded as myodegeneration as it also involves interstitial inflammation, edema, necrosis and accumulation of inflammatory cells. All of these breast muscle myopathies have been reported to be associated with fast muscle growth, the inadequate blood supply for increased muscle mass, and oxidative stress, consequently leading to tissue degeneration. Extensive muscle growth induced by muscle hypertrophy normally requires more blood supply but studies have observed that capillary density reduces with extensive muscle growth leading to hypoxia and poor transportation of waste products in muscles (Kuttappan et al., 2012 a; Papah et al., 2018; Sihvo et al., 2018; Soglia et al., 2016). Despite the extensive studies that have been conducted in recent years, the accurate cause of these myopathies is still an enigma. Unfortunately, the huge negative impact of these muscular anomalies on the sensory, technological and nutritional quality, and customer approval of breast meat, has been underscored. Therefore, a deep understanding of the fundamental causes and the search for permanent solutions are the need of the hour (Baldi et al., 2018; Soglia et al., 2016). This review discusses all significant information regarding characteristics, potential causes of BMM, relation with meat quality, and customer attitude toward these muscle abnormalities in chicken and provides direction to tackle the challenges the poultry industry faces.

\section{Major breast muscles myopathies in chicken}

Rapid strides of genetic improvement, better management and innovative nutritional approach over the last five decades have shown significant improvement in broiler muscle growth. This intensive increase in poultry production has solved food security up to much extent compared to the past. At the same time, it has introduced some new muscle abnormalities triggering the issue of meat quality (Huang and Ahn, 2018). With the rise of the poultry industry, some severe muscle abnormalities have been witnessed, including pale, soft, and exudative condition, green muscle disease (deep pectoral myopathy), white striping, woody breast, spaghetti meat, and dark, firm, and dry meat (DFD) having serious undesired consequences on meat quality, challenging consumer's preference (Ferreira et al., 2014; Guo and Dalrymple, 2017; Petracci et al., 2019). Some abnormalities were known long ago, like PSE and DPM. In contrast, other meat abnormalities have been observed in recent years of extensive production, including WB, WS and spaghetti meat-like conditions (Petracci et al., 2019; Zampiga et al., 2019). Most of these meat quality issues are related to the pectoralis major muscle. Recently, a massive increase in meat abnormalities has been reported in differ- ent parts of the world, with an estimated economic loss of up to \$1 billion per year to the United States' poultry industry (Petracci et al., 2019). These myopathies have severe impacts on the nutritional, functional, and sensory quality of meat, posing a potential threat to the broiler chicken industry. Figure 1 illustrates the physical changes in breast muscle of broiler affected with major muscle abnormalities, including WB, DPM, SM and WS.

\section{Wooden breast (WB)}

Recently, an emerging myopathy related to the major pectoral muscle causing the hardness of breast muscle has become a major challenge for the poultry industry. WB is a myodegenerative disease characterized by protruding ventral part of the major pectoral muscles, a layer of viscous fluid, white striations on muscle fibers with dotted hemorrhages hard consistency (Sihvo et al., 2014). Sihvo et al. (2017) studied the chicken breast affected from WB and found multifocal degeneration, muscle necrosis, absence of cross striations, infiltration of inflammatory cells in muscle fibers, perivascular infiltration, vasculitis and thickened perimysium connective tissue. Some studies also observed that WB changes the physical structure (shape, size and diameter) of muscle fiber. Physical characteristics of wooden breast muscles depend on the severity of the disease, including color change, the number of hemorrhages, the extent of hardness, and gelatinous fluid on breast muscles (Kuttappan et al., 2016; Tijare et al., 2016). Histopathological studies on wooden breast reported extensive fibrosis accompanying fat deposition and atrophic muscle fibers with polyphasic degeneration. The presence of more adipose tissues and connective tissues is one of the primary reasons behind the hardening of muscles (Kuttappan et al., 2016). WB leads to substantial economic losses to poultry farmers around the globe. A study on the etiology of WB unveiled that low protein content and higher deposition in breast muscles are the leading cause of wooden breast development. WB manifests multiple negative characteristics like low water holding capacity, significant cooking loss, and a higher exudate level in muscles (Sihvo et al., 2014; Soglia et al., 2018).

\section{White striping (WS)}

The incidence of WS in broiler has increased manifold for the last few years, disturbing the physical characteristics of major pectoral muscles leading to poor meat quality. In this disease, major pectoral muscles manifest white lines or stripes in the direction parallel to the skeletal muscle fibers (Mudalal et al., 2015). In short, major histological studies of WS affected muscles have revealed increased fat deposition, muscle fiber necrosis, interstitial inflammation accompanied by macrophages and lymphocytes infiltration (Soglia et al., 2016). The number and size of white stripes are solely dependent on the bird's strain and the severity of the condition. The severity of white stripes in skeletal muscles is divided into three phases (mild, moderate, and severe). The his- 
topathological study reported increased infiltration of lymphocytes and macrophages, absence of cross striations, mineral deposition in muscle cell nuclei, fibrosis, and lipidosis in muscles suffering from WS. In case of continuous stimuli or stress, permanent damage occurs to skeletal muscle satellite cells by converting into fibroblasts and adipocytes, leaving no chance for reparation. According to a study, more than $50 \%$ of broiler birds express WS in their pectoral muscles from days 59-63 of their age (Kuttappan et al., 2009). Another study showed that a physical examination of breast fillets from broiler chicken unveiled $74.5 \%$ of broiler birds were suffering from WS (Trocino et al., 2015).

In comparison, histopathological studies found white striped muscle fibers in $97 \%$ of birds (Trocino et al., 2015). Studies on chicken meat quality reported a dramatic increase in white striping incidence from $12 \%$ in 2012 (Petracci et al., 2013) to 29\% in 2015 (Russo et al., 2015), up to $75 \%$ in 2017 . This drastic increase in the incidence of white-striping meat has severe implications for better meat quality. A comparative study in two different broiler strains demonstrated that white striping's severity and incidence vary within different birds depending on the genetic line. The incidence of severe WS was reported much greater in Ross 308 (25.9\%) as compared to Cobb 500 (7.41\%) (Lorenzi et al., 2014).
Genetically modified heavy breeds reared in an intensive environment for commercial purposes had WS conditions in $40 \%$ of the total population (Lorenzi et al., 2014).

\section{Deep pectoral muscle myopathy (DPM)}

DPM is a degenerative muscle disorder called green muscle disease because it changes normal muscle color into green. DPM is characterized by the ischemic necrosis of minor pectoral muscles due to lack of activity in the respective muscle (Bailey et al., 2015; Bianchi et al., 2006). In birds, the pectoral region has two muscles, pectoralis major and pectoralis minor, both working together to move their wings. Pectoralis minor muscle is present between the sternum and pectoral major; it is sandwiched between two significant structures. When birds move their wings, it increases the pressure in the pectoral region's blood vessels by increasing blood flow towards pectoral muscles. In heavy commercial broiler breeds, the pectoralis major muscle grows extensively and puts pressure on the pectoralis minor. As pressure increases in the pectoral region, it stops the blood supply to pectoralis minor muscles by blocking blood vessels in that region (Jordan and Pattison, 1998). The blockage of blood vessels induces oxygen deficiency in minor pectoral muscles leading to cell death.
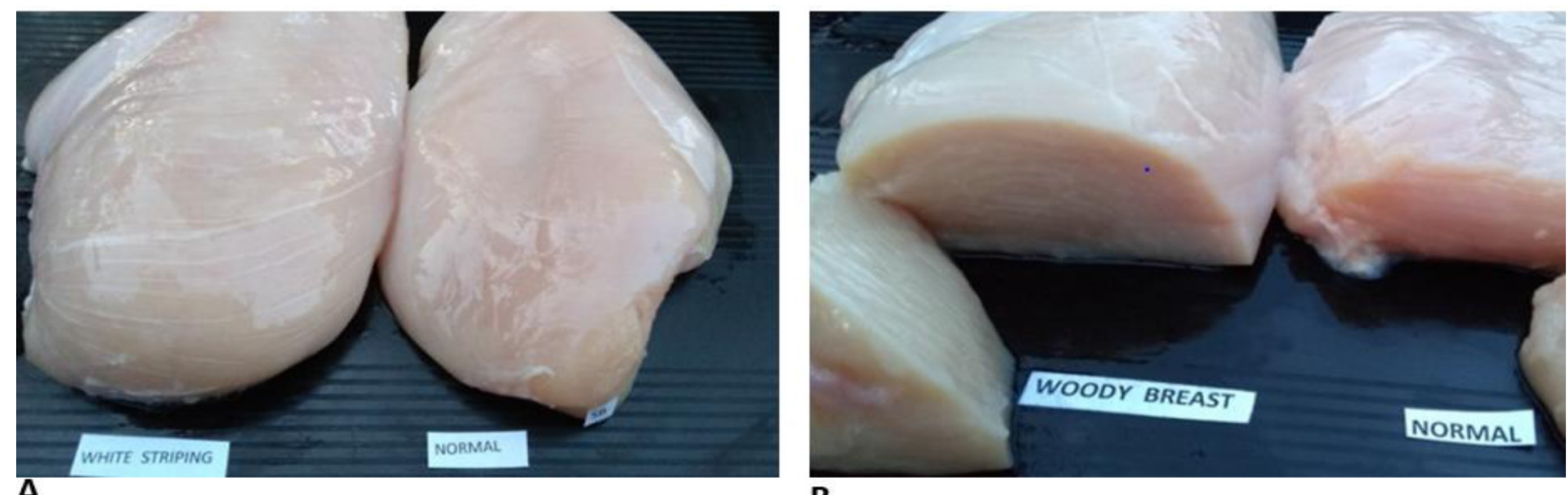

A

B
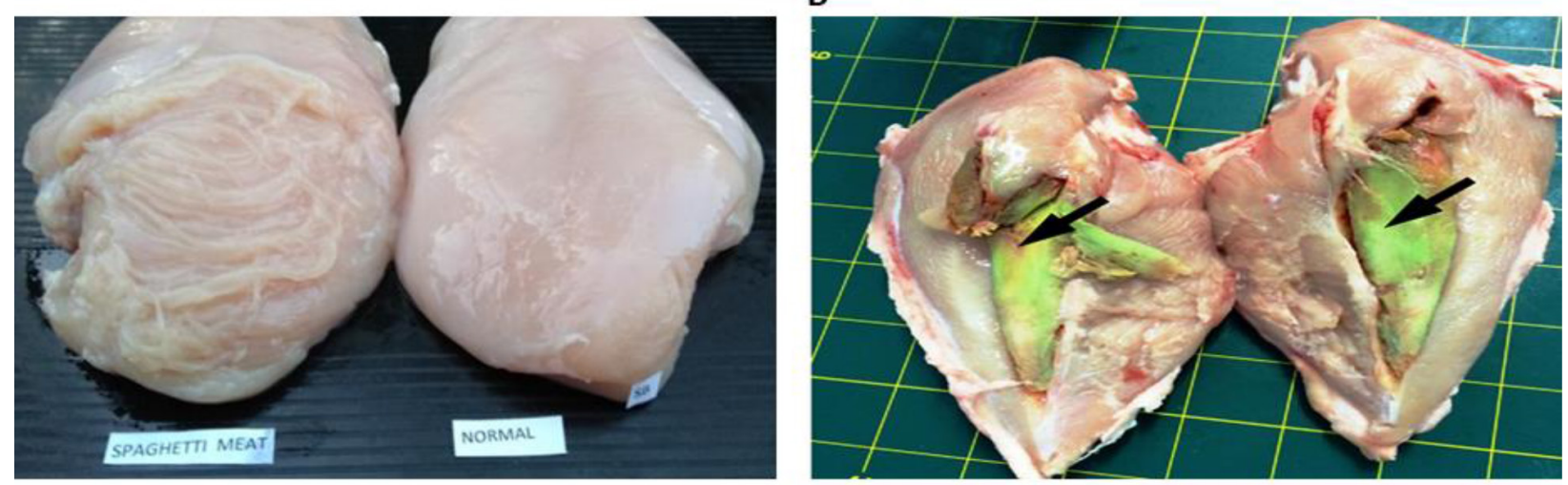

C

D

Figure 1. (A) White striping on chicken breast, white lines are more visible. (B) Wooden breast (WB) showing hemorrhages, showing white stripes hemorrhages and yellowish viscous fluid. (C) Spaghetti meat, disintegrated muscle fibers can be seen clearly. (D) DPM showing muscle damage and hemorrhages, the greenish part is much visible, indicating DPM (Barbut, 2019) 
The color of the muscles also changes from pink to greenish (Bilgili, 2016). During the early DPM period, muscles exhibit edema, inflammation, and hemorrhages; in later stages, the condition worsens with the onset of necrosis, muscle atrophy, immune cell infiltration, and increased connective tissues, and fibrosis (Kijowski and Kupińska, 2012). DPM incidence in chicken and turkey varies among birds depending on the birds' age, breed, and genotype. Chances of getting DPM are higher at a younger age ( $0-12 \%)$ as compared to older age (0-5.6\%) (Bianchi et al., 2006). A study reported a higher DPM incidence in Ross 308 (1.27\%) than Cobb500 (0.35\%). $3-17 \%$ more chances of spontaneous DPM were observed in broilers having higher growth rates (Bianchi et al., 2006). After slaughtering the birds having DPM, the breast portion with DPM needs to be removed, which poses severe economic loss to the poultry industry (Kijowski et al., 2014).

\section{Spaghetti meat}

Spaghetti meat is widely known as mushy breast, weak intramuscular connective tissues threadlike and detached muscle fiber bundles characterize this condition. Connective tissues in muscles are responsible for keeping the muscle fibers tightly bound together (Maiorano, 2017). The collagen present in connective tissues is necessary to maintain the integrity of muscle tissues. Immature connective tissues make muscles more friable and weak, and meat can easily be pulled apart by hand (Zampiga et al., 2019). In spaghetti meat conditions, connective tissue layers become weak, consequently following collapsed muscle fibers. Histological studies on SM reported similar lesions like WB and WS with an extensive proliferation of fatty tissues in endomysium and perimysium spaces of intramuscular connective tissues. However, the differentiating trait of SM is a progressive rarefaction of perimysium and endomysium connective tissues that damages structural integrity and compromises the cohesion among muscle fiber bundles. This condition is mostly seen in birds with high growth rates as perimysium septa is very weak in fast-growing broilers' pectoral muscles compared with other birds with slower growth rates (Bilgili, 2016; Radaelli et al., 2017; Sihvo et al., 2014).

The underlying mechanism in the development of breast muscle myopathies

Insufficient blood supply to increased muscle mass

In the past decade, numerous studies were conducted to find the underlying mechanism or pathway associated with the development of breast myopathies in chicken. However, the exact cause of the development of these muscle defects is not fully understood. Genetic selection for meat yield has been carried out extensively to fulfill the increasing global demands for chicken meat. These genetic selection strategies paid off with outstanding growth rates. Despite increased production efficiency, these genetic programs have changed the muscle architecture by increasing the diameter, length and number of myofiber, consequently reducing the density of blood supply in muscles (Hoving-Bolink et al., 2000). Consistently, studies conducted on commercial broiler strains have revealed that pectoral muscles are entirely comprised of anaerobic type IIB fibers, which corroborates the hypothesis that this condition makes pectoral muscles more susceptible to myopathies. Therefore, an increase in the size of fast-twitch muscle fiber through hypertrophy without improving the existing blood supply (capillary network) is the main culprit for these myopathies. As type IIB muscle fibers get energy from glycogen, during the metabolism of glycogen breakdown, lactic acid is being produced (Petracci et al., 2017). The circulatory system within myofibers is responsible for nutrient provision and removal of waste products like lactic acid. Through blood, lactic acid is transported to the liver, recycled, converted into glycogen and then sent back to circulation as an energy source (Sihvo et al., 2018). Thus, overall damage to breast muscles in fast-growing broilers is considered a result of a reduction in satellite cell number and in their altered abilities to proliferate and differentiate (Clark and Velleman, 2017; Daughtry et al., 2017). In commercial broiler strains, high muscle growth is achieved through hypertrophy, increased muscular hypertrophy reduces connective tissue spacing and leaves limited area for blood supply in muscle fibers leading to reduced capillary network. So, extreme muscle growth and reduced density of blood supply make oxygen transportation more difficult and also impair the mechanism to remove waste products from muscle tissues that ultimately damages the muscle (Alnahhas et al., 2016). All of these findings suggest that the physical changes in the muscle architecture linked to genetic selection for rapid growth and higher yield are predominantly related to the occurrence and severity of pectoral myopathies (Griffin et al., 2018).

Hypoxia and poor nutrient transportation in muscles

As all of the breast myopathies almost exhibit similar histological lesions, it is hypothesized that a common mechanism is involved in developing these defects. Fast growth and higher breast yield in commercial broiler are possible because of muscle fiber hypertrophy, which results in compromised blood supply, consequently triggering tissue hypoxia. Increased development of major pectoral muscle could compress the pectoral artery, reduce oxygen level, and impair nutrient transportation in muscles. This is why the severity of histological lesions increases as we move from surface to inner sections of pectoral muscles affected with myopathies (Baldi et al., 2018; Zampiga et al., 2020). Poor blood circulation in highly developed muscle tissues induces reduced oxygen level and tissue swelling due to the accumulation of compounds having osmotic characteristics. As discussed earlier, the transportation of metabolic wastes is also carried out by blood. Simultaneously, low capillary density in positively grown muscle fibers leads to the displacement of metabolic wastes and leads to phlebitis and perivascular lipid infiltration (Papah et al., 2017; Sihvo et al., 
2017). A study performed by (Papah et al., 2017) found arteriosclerosis and atherosclerosis in the birds affected with WB. In this context, it has been suggested that to overcome oxygen deficiency, blood supply increases through the synthesis of nitric oxide that ultimately triggers oxidative stress, further exacerbating the tissue inflammation and myodegeneration. Osmotic imbalance caused by hypoxia and myodegeneration induces ultrastructural changes in muscle fiber, including dilated sarcoplasmic reticulum, mitochondrial swelling, vacuolation, citrate loss and mitochondrial hyperplasia. Moreover, imbalance in calcium homeostasis could lead to proteases and lipases, thus contributing to further protein degradation in muscle tissues. Excessive myodegeneration in breast muscles overtake the regenerative ability of the muscle, which ultimately results in fibrosis and lipidosis, worsening the severity of muscle abnormalities (Abasht et al., 2016; Boerboom et al., 2018; Petracci et al., 2017; Sihvo et al., 2018; Zambonelli et al., 2016). A graphical representation of the mechanism involved in developing major breast muscle myopathies has shown in Figure 2.

Role of genetics in development of breast myopathies Breast muscle myopathies result in enormous economic loss to the poultry industry, leading to the condemnation of whole carcasses or portions. Genomic selection for better growth rates and high breast yield has been directed as the primary cause of some researchers' myopathies. Many studies suggest that genetic selection for high breast meat production has triggered breast muscle myopathies (Alnahhas et al., 2016; Bailey et al., 2015). However, studies conducted on large purebred commercial broiler flocks indicated that though there is a genetic component to the myopathies, the non-genetic factors contribute to greater than $65 \%$ of the variance in the incidence of WS of breast muscle and more than $90 \%$ of the variance of the incidence of WB in broiler chicken. Moreover, the genetic relation between breast yield and breast myopathies has revealed that factors other than growth and production are also responsible for the incidence of breast myopathies (Bailey et al., 2015).

A study carried out on two different broiler strains with different selection histories for breast yield reported another non-genetic factor responsible for all the breast muscle myopathy traits. Comprehensive breeding objectives, comprising the traits related to production, welfare, adaptability, and better reproductive status, are essential to achieving balanced broiler production progress (Hocking, 2014; Kapell et al., 2012). This breeding strategy approach has had and will continue to have a potential progressive trend for the broiler industry. However, researchers observed that increased breast meat production consequently leads to a higher incidence of breast myopathies. Still, the association of genetics with these traits is very low, which diverts the researchers' attention to other non-genetic factors linked to these myopathies (Bailey et al., 2020; Lilburn et al., 2019). Thus, more research is needed to fully understand the underlying mechanism of the non-genetic components such as management and nutrition and how they affect muscle growth and development in commercial chicken.

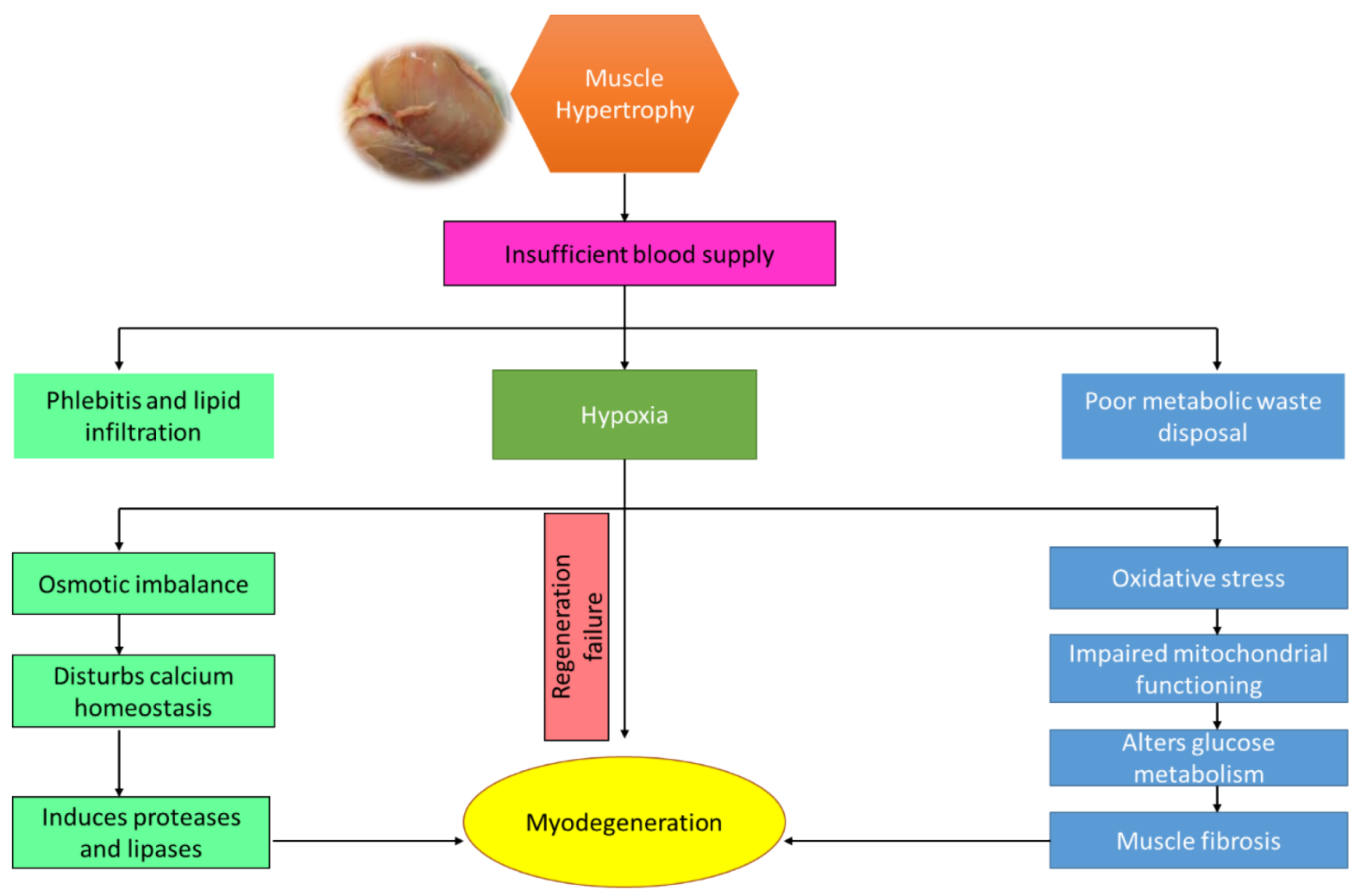

Figure 2. Flow chart diagram showing the mechanism involved in the development of breast muscle myopathies 
Incidence of breast myopathies is more in commercial fast growing chicken strains. Bailey et al. (2015) studied two commercial broiler strains and found the heritability of $0.024-0.097$ for WB and $0.185-0.338$ for WS that points that genetics also influences the development of myopathies but non-genetic factors also play a big role in this regard. The heritabilities for DPM, WB and SB are low with estimates ranging from 0.04 to 0.07 indicating a very strong influence of the non-genetic factors in the variation in manifestation of these myopathies. This specifies that selection only for body weight and breast muscle yield do not clarify the occurrence of the myopathies. Despite low to modest heritability for breast myopathies, Bailey et al. (2020) suggests that genetic factor plays a crucial role in the development of these myopathies and more studies are needed to fully comprehend the mechanism of how the myopathies initiate and develop. It has also been observed that reducing growth rate is linked to decreased incidence of myopathies but it affects the overall production performance of chicken. This issue can only be addressed by selecting and breeding the chickens according to the traits resistant to breast myopathies (Barbut, 2019).

\section{Effect of myopathies on meat quality in broiler}

Owing to a healthy nutritional profile, low cost and easy processing, a substantial increase in poultry meat demand has been noted since the past decades. This progressive trend in the poultry industry has led to rapid genetic improvement in poultry to develop fast-growing chicken to meet ever-growing poultry meat demands. All of the myopathies mentioned above affect meat quality, while the extent of quality deterioration depends on the severity of defects (Bilgili, 2016; Petracci et al., 2019). A study reported 200 million loss in the United States during 2016 just because of these abnormal muscle conditions as loss of a considerable portion of breast muscles because of myopathies account for substantial economic losses, which includes condemnation of whole breast/ carcass, reduced production, increased processing cost and customer rejection (Kuttappan et al., 2016).

\section{Myopathies damage the functional properties of chicken meat}

The functional characteristics of meat are associated with myofibrillar proteins' capacity to emulsify lipids, retain water and to form stable gels. Simultaneously, these proteins' quality depends on their structure, intricate fibrillary architecture, and amino acid composition (Pearce et al., 2011). Some biochemical processes in living cells, which mainly include proteolysis and oxidation, severely compromise proteins' ability to interact with other biomolecules such as lipids and water, consequently causing muscle defects. Among all breast muscle myopathies, the wooden breast exhibits a reduced capacity to hold water, low yield and marinade uptake, as multiple studies reported a substantial increase in drip loss and reduced marinade retention in the wooden breast as compared to normal breast muscle (Petracci et al., 2017; Sihvo et al., 2014; Trocino et al., 2015). According to previous studies, low muscle $\mathrm{pH}$ is responsible for poor water holding capacity in muscles (Dalgaard et al., 2018). Therefore, it is suggested that poor WHC in breast muscle myopathies is linked to the oxidative stress to proteins at the molecular level and severe muscle tissue degeneration. Research conducted by Chen et al. stated that PSE and WB meat have low gelation properties as compared to the standard meat, and adding $\mathrm{NaCl}$ from $1 \%$ to $4 \%$ could enhance the gel-forming ability of WB meat as $\mathrm{NaCl}$ improves the ionic strength and functionality of myofibrillar proteins (Chen et al., 2018). A nuclear magnetic resonance relaxation analysis of spaghetti meat unveiled the increased proportion of extra-myofibrillar water, which displayed a reduced WHC along with the presence of a higher amount of oxidized proteins. In short, these myopathies severely affect the protein functionality by impairing the myofibrillar structure through oxidative stress, ultimately triggering proteolysis (Baldi et al., 2018; Estévez, 2015, 2011).

\section{Myopathies directly influence meat's appearance, taste and texture}

The most important quality traits for poultry meat are appearance, texture and flavor. Appearance matters a lot during consumers' initial selection of the meat and the consumer's final satisfaction. The texture is the single most crucial sensory property for the quality assessment of meat (Carvalho et al., 2017; Estévez, 2015). The muscle tissue degeneration triggered by myopathies directly influences meat's sensory properties, including color, texture, and flavor. Breast muscle myopathies change the whole appearance of the breast as compared to normal breast muscle, and that is a direct consequence of histological changes during each myopathy characterized by visible white striations of multiple thicknesses in WS, bulging out, pale muscle fibers with hardened consistency in WB and collapsed/crumbled bundles of muscle fibers in SM. Multiple studies on the sensory properties of $\mathrm{WB}$ revealed increased lightness and yellowness in the color of WB meat. At the same time, these variations even got worse, depending on the severity of the condition (de Almeida Assunção et al., 2020; Tasoniero et al., 2016). Moreover, Zhuang and Bowker found that cooked WB was more dark, red and yellow than regular breast fillets (Zhuang and Bowker, 2018). Recently, it is observed that color modification is just superficial as a study found that color changes in WS and WB only occurred in the superficial muscle layer $(0.3 \mathrm{~cm})$ while the color of muscles was quite normal at deeper layers (Baldi et al., 2018). Soglia et al. (2017) tried to explain the different texture properties among superficial and deep layers by reporting that increased extracellular matrix, fibrosis, superficial layer having larger particles and higher compression force as compared to deep layer is responsible for color variation among superficial and deep layers (Soglia et al., 2018). Aguirre et al. (2018) found 
correspondence between texture profile analysis (TPA) and sensory evaluation of severe WB muscles (Aguirre et al., 2018). The muscles with myopathies displayed a higher level of hardness, cohesiveness, compactness, chewiness, springiness, crunchiness and fibrousness than normal chicken breasts. Consistently, Sanchez Brambila et al. (2016) found a higher level of cohesiveness and springiness in $\mathrm{WB}$ fillets cooked at $76^{\circ} \mathrm{C}$ than normal counterparts did (Sanchez Brambila et al., 2016). They also reported that springiness, hardness, and fibrousness were perceived differently between ventral and dorsal sections of cooked WB fillets. Unlike WB meat, the texture properties of meat during WS condition were not altered much. Many researchers reported negligible changes in the texture of chicken and turkey meat suffering from WS (Soglia et al., 2018). Only a few differences were found even in the most severe WS samples, and only trained panelists were able to differentiate the severe WS samples from normal ones (de Carvalho et al., 2020). Sanchez Brambila et al. (2016) clearly indicated that the WS condition does not affect the meat's textural properties, while the cooked breast fillets affected with WS exhibit a higher score for cohesiveness, hardness, and chewiness than normal breast fillets. Breast fillets affected with SM were observed to have a softer texture. This property displayed by SM is credited to the lower collagen content compared to normal meat and meat affected by WS and WB. Texture alteration in meat is not just linked to the collagen content, it is believed that muscle hardness during these myopathies possibly induced by several underlying mechanisms, which is not fully understood, and this aspect needs to be examined (Petracci et al., 2019; Zampiga et al., 2019).

\section{Myopathies disturb nutritive value of poultry meat}

From a human health point of view, when compared to other types of meat, it is verified that fat content in poultry meat is relatively low $(2.8 \mathrm{~g} / 100 \mathrm{~g}$ breast and $13 \mathrm{~g} / 100 \mathrm{~g}$ thigh) and with a positive unsaturated/saturated fatty acid ratio, thus making it a healthy food. Unfortunately, breast myopathies disturb the average nutritive value of poultry meat as it increases the number of fat contents and displays fewer protein contents than normal chicken breast (Baldi et al., 2019; Petracci et al., 2014). The respective change in meat composition enhances energy contents in breast meat suffering from myopathies $(451 \mathrm{Kj} / 100 \mathrm{~g})$ than in normal ones $(421 \mathrm{Kj} / 100 \mathrm{~g})(\mathrm{Pe}-$ tracci et al., 2014). No significant nutritional change has been reported in breast muscles myopathies; nutritional indexes suggest that lipid quality is the same between both types of breast meat, but a negligible variation in fatty acid composition has been reported. Breast myopathies mainly deteriorate meat quality by disturbing the protein contents, which includes degradation of myofibrillar proteins and increased collagen contents, oxidation of the muscle proteins and protein carbonylation (Estévez, 2011). Protein carbonylation is responsible for the consequent formation of insoluble aggregates and is linked to an impaired in vitro digestibility of oxidized protein (Estévez, 2015). To conclude, from previous studies, it is clear that breast myopathies in chicken have been linked with a higher level of lipid and increased protein oxidation, which is being identified as harmful for meat quality.

\section{Consumer behavior regarding myopathies}

Since consumers are the ultimate poultry meat recipients, their attitude towards these myopathies should be regarded as a relevant issue. The sole purpose of broiler production is to fulfill the consumer's demand regarding meat quality and nutritional values (Lusk, 2018). The broiler industry's focus is recently to increase the broiler muscle yield by selecting the fast-growing birds, subsequently increased the chances of myopathies severely compromise the consumer's expectations (Petracci et al., 2019). Myopathies affect the meat appearance, including petechial hemorrhages, excessive marbling, bulges and disintegration of muscles; all of these visual abnormalities contribute to instant meat rejection. A study conducted by Kuttappan et al. (2012 b) on consumer behavior towards breast meat affected with WS revealed that many participants recognized the growth abnormalities visually in severe WS condition. All the participant consumers identified the white striping as abnormal marbling that reduced acceptability and purchase desire. The author also reported an interesting phenomenon that 1 out of 5 consumers straightforwardly refused to eat the samples upon receiving awareness about myopathy, which shows consumers' emotional and behavioral response towards the myopathies. It is consumers' prerogative to know every information about the food they eat, and it could seriously compromise the acceptability, purchase intent and emotions evoked during the consumption of that product. Another study on consumer preferences reported gradual decrease in consumers' willingness as the severity of breast myopathy increased (6.9, normal; 6.1, moderate; 4.5, severe) (Kuttappan et al., 2012 b). Broadly speaking, almost $57 \%$ of the customers rejected the meat fillets with severe WS, while purchase intent also declined significantly. On further explanations, consumers revealed the reasons behind their rejection, which included higher marbling and abnormal color of the breast fillet.

\section{Strategies to reduce the chances of myopathies in broiler}

The production performance and health of birds or other livestock animals are mainly linked to the environmental status and management strategies. Consequently, the development of breast muscle myopathies is inherently linked to nutritional and environmental factors. Many types of research have been carried out to determine the possible factors involved in the occurrence of breast muscle myopathies in fast-growing broilers. It is widely accepted that the chances of breast abnormalities rise with higher body weight gain (Lorenzi et al., 2014; Papah et al., 2017), growth rate (Kuttappan et al., 2017; 
Lorenzi et al., 2014) and genetic modification for higher breast meat yield (Alnahhas et al., 2016; Livingston, 2018; Trocino et al., 2015), which is in complete agreement with strong genetic correlations found by Alnahhas et al. (2016). Histological studies suggested that the process of muscle degeneration during the development of myopathies is strictly associated with the age of birds (Griffin et al., 2018; Kawasaki et al., 2018; Papah et al., 2017) and breast growth pattern. It was recently demonstrated that egg storage duration before hatching and manipulation of embryonic development by egg incubation temperatures and chick weight at hatching could affect muscle morphology traits and related to breast abnormalities (Clark et al., 2017; Livingston, 2018). Ideal muscle growth is dependent on the functioning of the satellite cells within the muscle tissue. Satellite cells are specialized cells; their proliferation is at a peak during the first week after hatching and then declines; these cells play a crucial role in muscle fiber growth and repair. Inadequate satellite cell population in the muscle can badly affect the ability of a muscle to grow appropriately. In a low satellite cell population, muscles do not have the support needed for growth as the bird ages and this increases the chances of developing myopathies. It has been established that satellite cell population and activity are dependent on incubation conditions and the early management of the broiler chick. Delayed feeding or insufficient amino acid provision has been suggested to reduce the satellite cell population and increase fat deposition in the muscle. Therefore, better environmental and nutritive management of the modern broiler chicken is essential for optimum performance and muscle growth (Baldi et al., 2019; Petracci et al., 2019).

A study reported an increase in myopathies when broiler chickens were fed with diets containing low-quality fats, high amino acid density and low quantity of antioxidant nutrients, which displayed that faulty nutrition is the critical factor in developing breast muscle myopathy. The use of innovative nutritional approaches during key stages of broiler growth can considerably reduce myopathies' incidence and severity. Bird management has also been indicated as an essential factor in farm strategies to minimize breast myopathies' chances. Data gathered from different commercial broiler populations in the field has shown that insufficient ventilation leads to an increased carbon dioxide level in the shed, which is a potential risk factor for myopathies' development. This finding agrees with gene expression studies showing that myopathic muscles suffer from hypoxia, which may be linked to the farm conditions. While oxygen is not essential for the muscle fibers' activity in breast muscle (as they are predominantly anaerobic glycolytic), an adequate oxygen supply to the muscles is required as the satellite cells, and their associated cells require oxygen to fulfill their role (Lorenzi et al., 2014; Trocino et al., 2015).

The main strategy to reduce myopathies' occurrence includes reducing energy intake by either feed restric- tion or modifying feed formulation (Cruz et al., 2017; Livingston, 2018; Zampiga et al., 2019). However, an overall reduction in the occurrence of breast muscle abnormalities seems to be associated with reduced growth rate, body weight, and breast yield (Livingston, 2018). A study reported that dietary feed restriction and removing lysine from the diet have slightly reduced the severity of breast myopathies, especially WS and WB, but adopting these strategies under commercial conditions is not economically feasible. While a minor reduction in WB has been noted after dietary supplementation of guanidinoacetic acid (Córdova-Noboa et al., 2018), an increase of arginine: lysine ratio, the diet also has a mitigation effect on breast meat abnormalities (Zampiga et al., 2020). Therefore, until now, it appears that there is an absence of practical nutrition and management involvements to decrease growth-related abnormalities in the broiler industry without negatively affecting live and slaughter performances.

\section{Concluding remarks}

Setting a standard for a better quality of chicken meat is an arduous task as meat quality is indefinite and is not consistent with any defined description. It depends on several external and internal factors, but the most significant aspect is addressing the customer's demands. Actually, no reliable solutions have been devised yet to address the issue of breast muscle abnormalities in chicken. Therefore, to prevent the high chances of such myopathies is still an enigma for poultry researchers. A thorough understanding of molecular mechanisms and pathways involved in the pathogenesis of muscle myopathies is essential to provide an effective solution for this problem. The application of innovative technologies accompanied by an omics platform could be useful to provide reliable strategies. Metabolomics and proteomic studies in breast muscles suffering from abnormalities are the best way to get inner insight into the mechanisms involved in myopathies, which will further help develop diagnostic techniques. More studies are required to check the relationship between genetic selection and myopathies by comparing myopathies' incidence among the local and commercial broiler strains. As consumers' awareness of these chicken defects' incidence and basis is growing, converting abnormal breasts into processed products could be a feasible option. However, means to improve their technological, sensory, and nutritional characteristics are also much needed.

\section{Acknowledgment}

This work was supported by the Natural Science Foundation of China (31672412 and 31972550) and Guangdong Province (2020A1515011576). 


\section{References}

Abasht B., Mutryn M.F., Michalek R.D., Lee W.R. (2016). Oxidative stress and metabolic perturbations in wooden breast disorder in chickens. PLoS One, 0153750.

Aguirre M.E., Owens C.M., Miller R.K., Alvarado C.Z. (2018). Descriptive sensory and instrumental texture profile analysis of woody breast in marinated chicken. Poultry Sci., 97: 1456-1461.

Alnahhas N., Berri C., Chabault M., Chartrin P., Boulay M., Bourin M.C., Bihan-Duval E. Le (2016). Genetic parameters of white striping in relation to body weight, carcass composition, and meat quality traits in two broiler lines divergently selected for the ultimate $\mathrm{pH}$ of the pectoralis major muscle. BMC Genet., 17: 61.

Bailey R.A., Watson K.A., Bilgili S.F., Avendano S. (2015). The genetic basis of pectoralis major myopathies in modern broiler chicken lines. Poultry Sci., 94: 2870-2879.

Bailey R.A., Souza E., Avendano S. (2020). Characterising the influence of genetics on breast muscle myopathies in broiler chickens. Front. Physiol., https://doi.org/10.3389/fphys.2020.01041

Baldi G., Soglia F., Mazzoni M., Sirri F., Canonico L., Babini E., Laghi L., Cavani C., Petracci M. (2018). Implications of white striping and spaghetti meat abnormalities on meat quality and histological features in broilers. Animal, 12: 164-173.

Baldi G., Soglia F., Laghi L., Tappi S., Rocculi P., Tavaniello S., Prioriello D., Mucci R., Maiorano G., Petracci M. (2019). Comparison of quality traits among breast meat affected by current muscle abnormalities. Food Res. Int., 115: 369-376.

Barbut S. (2019). Recent myopathies in broiler's breast meat fillets. World. Poultry Sci. J., 75: 559-582.

Bianchi M., Petracci M., Franchini A., Cavani C. (2006). The occurrence of deep pectoral myopathy in roaster chickens. Poultry Sci., 85: $1843-1846$.

Bilgili S. (2016). Breast muscle abnormalities in broiler chickens. Am. Assoc. Avian Pathol., 5 pp.

Boerboom G., Van Kempen T., Navarro-Villa A., Pérez-Bonilla A. (2018). Unraveling the cause of white striping in broilers using metabolomics. Poultry Sci., 97: 3977-3986.

Carvalho R.H., Ida E.I., Madruga M.S., Martínez S.L., Shimokomaki M., Estévez M. (2017). Underlying connections between the redox system imbalance, protein oxidation and impaired quality traits in pale, soft and exudative (PSE) poultry meat. Food Chem. 215: 129-137.

Chen H., Wang H., Qi J., Wang M., Xu X., Zhou G. (2018). Chicken breast quality - normal, pale, soft and exudative (PSE) and woody - influences the functional properties of meat batters. Int. J. Food Sci. Technol., 53: 654-664.

Clark D.L., Velleman S.G. (2016). Spatial influence on breast muscle morphological structure, myofiber size, and gene expression associated with the wooden breast myopathy in broilers. Poultry Sci. 95: 2930-2945.

Clark D.L., Walter K.G., Velleman S.G. (2017). Incubation temperature and time of hatch impact broiler muscle growth and morphology. Poultry Sci., 96: 4085-4095.

Córdova-Noboa H.A., Oviedo-Rondón E.O., Sarsour A.H., Barnes J., Ferzola P., Rademacher-Heilshorn M., Braun U. (2018). Performance, meat quality, and pectoral myopathies of broilers fed either corn or sorghum based diets supplemented with guanidinoacetic acid. Poultry Sci., 97: 2479-2493.

Cruz R.F.A., Vieira S.L., Kindlein L., Kipper M., Cemin H.S., Rauber S.M. (2017). Occurrence of white striping and wooden breast in broilers fed grower and finisher diets with increasing Lysine levels. Poultry Sci., 96: 501-510.

Dalgaard L.B., Rasmussen M.K., Bertram H.C., Jensen J.A., Møller H.S., Aaslyng M.D., Hejbøl E.K., Pedersen J.R., Elsser-Gravesen D., Young J.F. (2018). Classification of wooden breast myopathy in chicken pectoralis major by a standardised method and association with conventional quality assessments. Int. J. Food Sci. Technol., https://doi.org/10.1111/ijfs.13759

Daughtry M.R., Berio E., Shen Z., Suess E.J.R., Shah N., Geiger A.E., Berguson E.R., Dalloul R.A., Persia M.E., Shi H., Gerrard D.E. (2017). Satellite cell-mediated breast muscle regeneration decreases with broiler size. Poultry Sci., 96: 3457-3464. de Almeida Assunção A.S., Garcia R.G., Komiyama C.M., de Sena Gandra É.R., de Souza J.R., dos Santos W., Caldara F.R., Martins R.A. (2020). Wooden breast myopathy on broiler breast fillets affects quality and consumer preference. Trop. Anim. Health Prod., 52: 3555-3565.

de Carvalho L.M., Ventanas S., Olegario L.S., Madruga M.S., Estévez M. (2020). Consumers awareness of white-striping as a chicken breast myopathy affects their purchasing decision and emotional responses. LWT, 131: 109809.

Estévez M. (2011). Protein carbonyls in meat systems: A review. Meat Sci., 89: 259-279.

Estévez M. (2015). Oxidative damage to poultry: from farm to fork. Poultry Sci., 94: 1368-1378.

Ferreira T.Z., Casagrande R.A., Vieira S.L., Driemeier D., Kindlein L. (2014). An investigation of a reported case of white striping in broilers. J. Appl. Poultry Res., 23: 748-753.

Griffin J.R., Moraes L., Wick M., Lilburn M.S. (2018). Onset of white striping and progression into wooden breast as defined by myopathic changes underlying Pectoralis major growth. Estimation of growth parameters as predictors for stage of myopathy progression. Avian Pathol., 47: 2-13.

Guo B., Dalrymple B.P. (2017). Transcriptomics of meat quality. In: New aspects of meat quality. Elsevier, pp. 259-320.

Hocking P.M. (2014). Unexpected consequences of genetic selection in broilers and turkeys: problems and solutions. Brit. Poultry Sci., 55: $1-12$.

Hoving-Bolink A.H., Kranen R.W., Klont R.E., Gerritsen C.L.M., de Greef K.H. (2000). Fibre area and capillary supply in broiler breast muscle in relation to productivity and ascites. Meat Sci., 56: $397-402$.

Huang X., Ahn D.U. (2018). The incidence of muscle abnormalities in broiler breast meat - a review. Korean J. Food Sci. Anim. Resour., 38: 835-850.

Jordan F.T.W., Pattison M. (1998). Deep pectoral myopathy of turkeys and chickens. In Poultry diseases. Saunders Elsevier, London, UK, pp. 398-399.

Kapell D.N.R.G., Hill W.G., Neeteson A.-M., McAdam J., Koerhuis A.N.M., Avendaño S. (2012). Twenty-five years of selection for improved leg health in purebred broiler lines and underlying genetic parameters. Poultry Sci., 91: 3032-3043.

Kawasaki T., Iwasaki T., Yamada M., Yoshida T., Watanabe T. (2018). Rapid growth rate results in remarkably hardened breast in broilers during the middle stage of rearing: A biochemical and histopathological study. PLoS One, 13: e0193307.

Kijowski J., Kupińska E. (2012). Induction of DPM changes in broiler chickens and characteristics of myopathy symptoms. Bull. Vet. Inst. Pulawy, 56: 217-223.

Kijowski J., Kupińska E., Stangierski J., Tomaszewska-Gras J., Szablewski T. (2014). Paradigm of deep pectoral myopathy in broiler chickens. World. Poultry Sci. J., 70: 125-138.

Kuttappan V.A., Brewer V.B., Clark F.D., Mckee S.R., Meullenet J.F., Emmert J.L., Owens C.M. (2009). Effect of white striping on the histological and meat quality characteristics of broiler fillets. Poultry Sci., 88.

Kuttappan V.A., Brewer V.B., Apple J.K., Waldroup P.W., Owens C.M. (2012 a). Influence of growth rate on the occurrence of white striping in broiler breast fillets. Poultry Sci., 91: 2677-2685.

Kuttappan V.A., Lee Y.S., Erf G.F., Meullenet J.F.C., Mckee S.R., Owens C.M. (2012 b). Consumer acceptance of visual appearance of broiler breast meat with varying degrees of white striping. Poultry Sci., 91: 1240-1247.

Kuttappan V.A., Hargism B.M., Owens C.M. (2016). White striping and woody breast myopathies in the modern poultry industry: A review. Poultry Sci., 95: 2724-2733.

Kuttappan V.A., Bottje W., Ramnathan R., Hartson S.D., Coon C.N., Kong B.W., Owens C.M., Vazquez-A Non M., Hargis B.M. (2017). Proteomic analysis reveals changes in carbohydrate and protein metabolism associated with broiler breast myopathy. Poultry Sci., 96: 2992-2999.

Lilburn M.S., Griffin J.R., Wick M. (2019). From muscle to food: Oxidative challenges and developmental anomalies in poultry breast muscle. Poultry Sci., 98: 4255-4260. 
Livingston M.L. (2018). Physiological and nutritional aspects of broiler breast myopathies. ProQuest Diss. Theses., 10969836.

Lorenzi M., Mudalal S., Cavani C., Petracci M. (2014). Incidence of white striping under commercial conditions in medium and heavy broiler chickens in Italy. J. Appl. Poultry Res., 23: $754-758$.

Lusk J.L. (2018). Consumer preferences for and beliefs about slow growth chicken. Poultry Sci., 97: 4159-4166.

Maiorano G. (2017). Meat defects and emergent muscle myopathies in broiler chickens: implications for the modern poultry industry. Sci. Ann. Pol. Soc. Anim. Prod., 13: 43-51.

Mudalal S., Lorenzi M., Soglia F., Cavani C., Petracci M. (2015). Implications of white striping and wooden breast abnormalities on quality traits of raw and marinated chicken meat. Animal, 9: 728-734.

Papah M.B., Brannick E.M., Schmidt C.J., Abasht B. (2017). Evidence and role of phlebitis and lipid infiltration in the onset and pathogenesis of Wooden Breast Disease in modern broiler chickens. Avian Pathol., 46: 623-643.

Papah M.B., Brannick E.M., Schmidt C.J., Abasht B. (2018). Gene expression profiling of the early pathogenesis of wooden breast disease in commercial broiler chickens using RNA-sequencing. PLoS One, 13: e0207346.

Pearce K.L., Rosenvold K., Andersen H.J., Hopkins D.L. (2011). Water distribution and mobility in meat during the conversion of muscle to meat and ageing and the impacts on fresh meat quality attributes - a review. Meat Sci., 89: 111-124.

Petracci M., Mudalal S., Bonfiglio A., Cavani C. (2013). Occurrence of white striping under commercial conditions and its impact on breast meat quality in broiler chickens. Poultry Sci., 92: 1670 1675.

Petracci M., Mudalal S., Babini E., Cavani C. (2014). Effect of white striping on chemical composition and nutritional value of chicken breast meat. Ital. J. Anim. Sci., 13: 3138.

Petracci M., Soglia F., Berri C. (2017). Muscle metabolism and meat quality abnormalities. In: Poultry Quality Evaluation. Elsevier, pp. $51-75$.

Petracci M., Soglia F., Madruga M., Carvalho L., Ida E., Estévez M. (2019). Wooden-breast, white striping, and spaghetti meat: causes, consequences and consumer perception of emerging broiler meat abnormalities. Compr. Rev. Food Sci. Food Saf., https://doi org/10.1111/1541-4337.12431

Radaelli G., Piccirillo A., Birolo M., Bertotto D., Gratta F., Ballarin C., Vascellari M., Xiccato G., Trocino A. (2017). Effect of age on the occurrence of muscle fiber degeneration associated with myopathies in broiler chickens submitted to feed restriction. Poultry Sci., 96: 309-319.

Russo E., Drigo M., Longoni C., Pezzotti R., Fasoli P., Recordati C. (2015). Evaluation of white striping prevalence and predisposing factors in broilers at slaughter. Poultry Sci., 94: 1843-1848.

Sanchez Brambila G., Bowker B.C., Zhuang H. (2016). Comparison of sensory texture attributes of broiler breast fillets with different degrees of white striping. Poultry Sci., 95: 2472-2476.
Sihvo H.-K., Immonen K., Puolanne E. (2014). Myodegeneration with fibrosis and regeneration in the pectoralis major muscle of broilers. Vet. Pathol., 51: 619-623.

Sihvo H.K., Lindén J., Airas N., Immonen K., Valaja J., Puolanne E. (2017). Wooden Breast myodegeneration of pectoralis major muscle over the growth period in broilers. Vet. Pathol., 54: 119-128.

Sihvo H.-K., Airas N., Lindén J., Puolanne E. (2018). Pectoral vessel density and early ultrastructural changes in broiler chicken wooden breast myopathy. J. Comp. Pathol., 161: 1-10.

Skaperda Z., Veskoukis A., Kouretas D. (2019). Farm animal welfare, productivity and meat quality: Interrelation with redox status regulation and antioxidant supplementation as a nutritional intervention (Review). World Acad. Sci. J., 1: 177-183.

Soglia F., Mudalal S., Babini E., Di Nunzio M., Mazzoni M., Sirri F., Cavani C., Petracci M., (2016). Histology, composition, and quality traits of chicken Pectoralis major muscle affected by wooden breast abnormality. Poultry Sci., 95: 651-659.

Soglia F., Baldi G., Laghi L., Mudalal S., Cavani C., Petracci M. (2018). Effect of white striping on turkey breast meat quality. Animal, 12: 2198-2204.

Tasoniero G., Cullere M., Cecchinato M., Puolanne E., Dalle Zotte A. (2016). Technological quality, mineral profile, and sensory attributes of broiler chicken breasts affected by White Striping and Wooden Breast myopathies. Poultry Sci., 95: 2707-2714.

Tijare V.V., Yang F.L., Kuttappan V.A., Alvarado C.Z., Coon C.N., Owens C.M. (2016). Meat quality of broiler breast fillets with white striping and woody breast muscle myopathies. Poultry Sci., 95: 2167-2173.

Trocino A., Piccirillo A., Birolo M., Radaelli G., Bertotto D., Filiou E., Petracci M., Xiccato G., (2015). Effect of genotype, gender and feed restriction on growth, meat quality and the occurrence of white striping and wooden breast in broiler chickens. Poultry Sci., 94: 2996-3004.

Wideman N., O’Bryan C.A., Crandall P.G. (2016). Factors affecting poultry meat colour and consumer preferences - a review. World. Poultry Sci. J., 72: 353-366.

Zambonelli P., Zappaterra M., Soglia F., Petracci M., Sirri F., Cavani C., Davoli R. (2016). Detection of differentially expressed genes in broiler pectoralis major muscle affected by White Striping Wooden Breast myopathies. Poultry Sci., 95: 2771-2785.

Zampiga M., Meluzzi A., Pignata S., Sirri F. (2019). Occurrence of breast meat abnormalities and foot pad dermatitis in light-size broiler chicken hybrids. Animals (Basel), 9: 706.

Zampiga M., Soglia F., Baldi G., Petracci M., Strasburg G.M., Sirri F. (2020). Muscle abnormalities and meat quality consequences in modern turkey hybrids. Front. Physiol., https://doi.org/10.3389/ fphys. 2020.00554

Zhuang H., Bowker B. (2018). The wooden breast condition results in surface discoloration of cooked broiler pectoralis major. Poultry Sci., 97: 4458-4461.

Received: 17 III 2021

Accepted: 20 IX 2021 